\title{
REVUE
}

\section{L'EXAMEN PAR LES SPÉCIALISTES ANGLAIS DE LA LAINE SYNTHÉTIQUE PRÉPARÉE EN PARTANT DE CASÉINE}

\author{
par \\ G. GÉNIN
}

La "Wool Industries Research Association" vient de publier le compte rendu des essais qui ont été effectués sur la nouvelle laine synthétique à base de caséine fabriquée en Italie. Ces essais ont été effectués sous la direction de M. B. H. WiLson.

Ce rapport indique que l'aspect extérieur de ce nouveau produit synthétique est assez voisin de celui de la laine mérinos avec cette différence toutefois que la fibre synthétique n'est pas aussi frisée et que son toucher donne une impression plus rugueuse. Si on effectue un examen microscopique, on constate une différence essentielle due à ce que la fibre synthétique ne possède pas de parties écaillées. Cette observation montre déjà qu'il serait peu probable que la laine de easéine puisse être utilisée pour la fabrication de tissus compacts et chauds.

Il faut d'ailleurs rappeler que cette nouvelle fibre synthétique n'est pas la première qui ait été faite en utilisant soit la caséine du lait, soit certaines protéines dénaturées, et comme toutes les fibres faites en partant de ces mêmes matières premières, on a constaté que la nouvelle laine synthétique contient une certaine proportion de formaldéhyde.

Il existe également un test qui permet d'apprécier la qualité d'une fibre et qui repose sur le fait que lorsque des fibres animales sont plongées dans une solution alcaline, elles sont, au point de vue chimique, plus ou moins modifiées et une portion de leurs constituants azotés passe en solution, de telle sorte qu'en déterminant l'azote soluble qui est passé dans l'extrait, on peut obtenir de cette façon une indication sur le degré d'altération de la fibre. On a constaté par cet essai que la dégradation subie par la laine de caséine est environ 10 fois plus grande que celle que supporte une laine naturelle plongée dans une solution d'une concentration cependant 5 fois plus grande.

On a également constaté, en comparant la résistance de la laine artificielle et celle de la laine naturelle à l'action de la trypsine, que les fibres synthétiques sont beaucoup moins résistantes que les fibres naturelles. 


\section{COMPARAISONS AVEC LA LAINE NAT URELLE}

La laine naturelle ainsi que le produit artificiel gonflent lorsqu'ils sont plongés dans l'eau. L'augmentation du diamètre de la fibre est du même ordre de grandeur pour les deux produits et d'environ $7 \%$. Par contre, le gonflement longitudinal est beaucoup plus important pour le produit synthétique et atteint $7,5 \%$ contre 1 à $2 \%$ pour le produit naturel. Si on soumet la fibre synthétique à un effort d'extension, alors qu'elle est plongée dans l'eau, on constate que, par suite de sa très grande plasticité, elle s'allonge considérablement, atteint souvent le double de sa longueur initiale et se brise sous une charge qui est comprise entre le quart et le huitième de celle qu'il faut pour rompre un fil de laine naturel de même diamètre.

Or, l'expérience a montré que la laine naturelle a une propriété essentielle, celle de conserver son élasticité lorsque la fibre est humide. Cette propriété présente une importance de tout premier ordre et des travaux récents ont montré qu'elle est due à l'orientation de molécules à longue chaîne qui se mettent parallèlement les unes aux autres lorsque la fibre est étirée, et qui au contraire reprennent leur position initiale de serpentin lorsque l'effort d'extension est annulé. La stabilité de ces chaînes, qu'elles soient à l'état de spirale ou à l'état étiré, est due à l'existence de liaisons entre chaînes voisines dont les plus importantes résultent de la présence du soufre dans la cystine. Ce serait à cette liaison que les propriétés essentielles de la laine sont dues.

La laine synthétique italienne à base de caséine est constituée de produits analogues à la cératine, mais ses molécules sont complètement mêlées entre elles; ces molécules ont bien tendance à se disposer les unes auprès des autres, mais dès que la fibre est mouillée, il suffit d'une très petite force pour étirer le filament, et de même une force très légère empêche la contraction de ce filament.

D'après le rapport anglais, cette nouvelle fibre synthétique ne pourrait servir qu'en mélange avec des fibres plus stables de coton ou de viscose, pour la fabrication de tissus spéciaux destinés à répondre à des besoins particuliers qui résultent de la situation économique italienne. Par contre, il semble très peu, vraisemblable que cette laine synthétique puisse remplacer la laine, sauf dans des cas tout à fait spéciaux.

$\mathrm{Au}$ point de vue teinture, la laine synthétique ne présente aucune caractéristique particulière, si ce n'est qu'elle a une très grande affinité pour les colorants acides. 